\title{
Base social da saúde e ponderação de contexto na contratualização
}

Paula Broeiro-Gonçalves ${ }^{1}$

A dissonância entre a contratualização nos cuidados de saúde primários (CSP), o desempenho das unidades de saúde e os resultados conduz a esta reflexão sobre a base social da saúde. ${ }^{1}$

No Relatório de Primavera de 2019 foram identificados alguns dos riscos da contratualização nos CSP: serem focados na doença e não na pessoa; excluírem a complexidade psicossocial; potenciarem a concentração desproporcionada de atenção e esforço dos profissionais em objetivos de curto prazo e/ou nos indicadores incentivados. ${ }^{2}$ As alterações à contratualização introduzidas em 2017 atenderam a algumas destas preocupações, das quais se realça a avaliação operacionalizada através de uma matriz multidimensional, o Índice de Desempenho Global (IDG), que conferiu sentido de flexibilidade. Apesar do empenho na definição da matriz de indicadores subsiste a utilização de instrumentos «imperfeitos», incapazes de avaliar holisticamente o desempenho numa atividade complexa, altamente dependente do contexto. ${ }^{2}$ Importa, assim, atender à base social da saúde global e integrá-la em todas as etapas da contratualização nos CSP.

A Organização Mundial da Saúde define como determinantes sociais de saúde (DSS) as "condições em que as pessoas nascem, crescem, vivem, trabalham e envelhecem [que] as quais são moldadas pela distribuição de rendimento, poder e recursos em níveis global, nacional e local". ${ }^{3}$ Essas condições influenciam a oportunidade de uma pessoa ser saudável, o risco de adoecer e a expectativa de vida. A Healthy People 2030, ao definir DSS, dá-lhes uma perspetiva longitudinal e ambiental, como "condições nos ambientes em que as

1. Médica de Família. UCSP dos Olivais, ACeS Lisboa Central. Lisboa, Portugal. pessoas nascem, vivem, aprendem, trabalham, brincam e envelhecem que afetam uma ampla gama de saúde e qualidade de vida". ${ }^{4}$ Habitualmente as iniquidades sociais em saúde - as diferenças injustas e evitáveis no estado de saúde entre os grupos da sociedade - são aquelas que resultam da distribuição desigual dos determinantes sociais. ${ }^{5-6}$

A base social da saúde refere-se ao peso dos fatores sociais e ambientais na saúde global do indivíduo, sabendo-se que na diversidade de fatores envolvidos o impacto dos fatores sociais e ambientais é muito superior (cerca de $80 \%$ ) ao dos cuidados de saúde (cerca de 20\%). ${ }^{1}$ Oferecer acesso universal ${ }^{7}$ a bons cuidados de saúde é, portanto, necessário, mas insuficiente para otimizar a saúde das populações e reduzir as iniquidades em saúde. ${ }^{8}$ Para melhorar a saúde, reduzir as desigualdades e reduzir os custos é preciso melhorar as condições em que as pessoas nascem, vivem, trabalham e envelhecem. ${ }^{8}$ Diderichsen explicita como a diferença na posição social é responsável pela iniquidade em saúde e se confunde com os determinantes sociais da saúde e com os processos sociais que moldam a distribuição desigual desses determinantes. ${ }^{9}$ Em síntese, mecanismos sociais, económicos e políticos dão origem a um conjunto de posições socioeconómicas, designado de gradiente social, em que a doença retroalimenta a posição social (e.g., doença mental, multimorbilidade). Existe maior probabilidade de ocorrerem mais doenças e mais graves em pessoas socialmente desfavorecidas. ${ }^{10}$

O gradiente social conhecido como o conjunto de posições que os indivíduos tomam na sociedade resulta do património transmitido no seio da família (disciplina, autocontrolo, pensamento prospetivo, respeito pelo espaço alheio). ${ }^{11}$ A pobreza, por exemplo, é um determinante da posição no gradiente social. A Associação Quarto Mundo, conjuntamente com a 
Universidade de Oxford, amplificou e expandiu a compreensão real do que significa a pobreza aos olhos de quem nunca a viveu. Uma das curiosidades que surgiu desta pesquisa sobre as dimensões ocultas da pobreza foi a transversalidade dessas dimensões entre culturas e países. ${ }^{12}$ Das nove dimensões da pobreza identificadas por este consórcio, a par das mais documentadas privações, como a falta de trabalho digno, rendimento insuficiente e inseguro, privação material e social, surgem seis outras dimensões ocultas:

- As que constituem a experiência central de pobreza, como o sofrimento no corpo/mente/coração; o desempoderamento e a luta e resistência.

- As relacionais, que refletem o modo como as pessoas que não estão em situação de pobreza afetam a vida daqueles que estão: maus-tratos sociais, maus-tratos institucionais e não reconhecimento de contri-

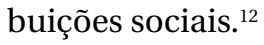

Destacam-se as dimensões centrais da pobreza que se refletem no processo de saúde e doença, como o desempoderamento ou falta de controlo nas escolhas. Viver na pobreza significa vivenciar uma intensa experiência de sofrimento físico, mental e emocional acompanhado por uma sensação de impotência. As vidas são, ainda, encurtadas, porque a saúde física e mental é prejudicada por habitação precária de baixa qualidade, dieta inadequada e lutas diárias pela sobrevivência. Muitas pessoas na pobreza não podem praticar cuidados preventivos porque não têm acesso a alimentos saudáveis, sendo a desnutrição e a obesidade prevalentes. Problemas de saúde causados por múltiplas privações levam a sequelas físicas e emocionais, que conduzem à autonegligência ou ao comportamento adito como alívio do sofrimento. As experiências diárias de injustiça e desumanização afetam a autoestima e a capacidade de mudar. ${ }^{12}$

Os determinantes sociais muitas vezes confundem o esforço dos CSP para melhorar a saúde dos seus doentes. Apesar da evidência disponível em torno da medicina e da prevenção, ambientes insalubres e desigualdades sociais resultam em estilos de vida não saudáveis, deficiente utilização de serviços de saúde e autocuidados, acentuando desigualdades em saúde. ${ }^{1}$

O sistema de saúde torna-se particularmente relevante através da questão do acesso e do seu poder de mediação das consequências da doença na vida das pessoas. Não se pode, no entanto, ignorar que os sistemas de saúde podem também contribuir para o agravamento da desigualdade, quando pessoas com as mesmas necessidades não têm acesso aos mesmos recursos, o que se designa por desigualdade horizontal. ${ }^{10} \mathrm{Em}$ contraponto, a desigualdade vertical significa que pessoas com maiores necessidades não dispõem de maiores recursos. Consequentemente, o que se considera equidade (uso igual em todos os subgrupos da população) é, na verdade, iniquidade. ${ }^{10}$ É, pois, necessária uma mudança de paradigma de cuidados de saúde, de centrados na doença para cuidados centrados na pessoa. ${ }^{10}$ De acordo com Barbara Starfield, a desigualdade está embutida nos sistemas de saúde - especialmente nos ocidentais, que subestimam os CSP ao basearem-se numa visão de necessidades de saúde centrada na doença e não na pessoa. Desperdiçam, por isso, a eficiência, o designado paradoxo dos CSP. Os CSP, quando comparados com os diferenciados, revelaram resultados semelhantes, com maior equidade e menor custo para "pessoas inteiras» e populações. ${ }^{13}$

O que terá que mudar? Terá que mudar a lente com que se observa a saúde global e, consequentemente, a organização dos cuidados e a contratualização.

Os determinantes sociais muitas vezes confundem o esforço dos CSP para melhorar a saúde da população que servem, sendo o investimento com maior retorno em saúde o efetuado nas condições de vida das pessoas. Operar uma mudança significativa na saúde da população pressupõe o desenvolvimento de trabalho colaborativo entre setores da sociedade para abordar os determinantes sociais e ambientais. ${ }^{1}$

Quanto à contratualização, por um lado, os reguladores deverão diminuir a sobrestimação do desempenho, dos serviços de saúde e dos seus profissionais nos resultados em saúde. Por outro lado, os próprios profissionais deverão aceitar o limite da sua intervenção e recentrar o modelo de contratualização em indicadores centrados na pessoa, com impacto na saúde, como o acesso e a qualidade de cuidados. A contratualização deverá, ainda, refletir o modelo biopsicossocial próprio dos CSP e espelhar a complexidade da realidade, sendo necessário que o IDG seja adequadamente ponderado/corrigido de acordo com o contexto. O grande salto qualitativo do IDG está, portanto, na capacidade de aumentar a sua «tolerabilidade» aos momentos do 
ciclo de vida da organização (e.g., ausência de profissionais) e adequar à especificidade da população, incluindo dimensões socioeconómicas (e.g., multiculturalidade, insuficiência económica). ${ }^{2}$

\section{REFERÊNCIAS BIBLIOGRÁFICAS}

1. Silberberg $M$, Martinez-Bianchi V, Lyn MJ. What is population health? Prim Care. 2019;46(4):475-84.

2. Pestana J, Dimitrovová K, Charondière $P$, Broeiro $P$, Cardoso S. Necessidade de um novo impulso na reforma dos cuidados de saúde primários: contrariar as desigualdades, integrar os contextos da prática e os determinantes sociais na análise dos desempenhos. In: Observatório Português dos Sistemas de Saúde, editor. Saúde - Um direito humano: relatório de Primavera 2019 [Internet]. Lisboa: OPSS; 2019. p. 35-69. Available from: http://opss.pt/wp-content/uploads/2019/07/ RP2019.pdf

3. Malamou T. Social determinants of health. Nosileftiki. 2015;54(3):23140.

4. Office of Disease Prevention and Health Promotion. Healthy people 2030: social determinants of health [homepage]. Washington: U.S. Department of Health and Human Services; 2017. Available from: https://health.gov/healthypeople/objectives-and-data/social-determinants-health

5. Davidson KW, Kemper AR, Doubeni CA, Tseng CW, Simon MA, Kubik M, et al. Developing primary care-based recommendations for social determinants of health: methods of the U.S. Preventive Services Task Force. Ann Intern Med. 2020;173(6):461-7.

6. Tamayo M, Besoaín Á, Rebolledo J. Determinantes sociales de la salud y discapacidad: actualizando el modelo de determinación [Social determinants of health and disability: updating the model for determination]. Gac Sanit. 2018;32(1):96-100. Spanish

7. Cu A, Meister S, Lefebvre B, Ridde V. Assessing healthcare access using the Levesque's conceptual framework: a scoping review. Int J Equity Health. 2021;20(1):116.

8. Donkin A, Goldblatt P, Allen J, Nathanson V, Marmot M. Global action on the social determinants of health. BMJ Glob Health. 2017;3: e000603.

9. Diderichsen F, Andersen I, Manuel C, Andersen AM, Bach E, Baadsgaard $M$, et al. Health inequality: determinants and policies. Scand J Public Health. 2012;40(8 Suppl):12-105.

10. Starfield B. The hidden inequity in health care. Int J Equity Health. 2011;10:15

11. Ferreira MA, Latorre MR. Desigualdade social e os estudos epidemiológicos: uma reflexão [Social inequality and epidemiological studies:a reflection]. Cienc Saude Colet. 2012;17(9):2523-31. Portuguese

12. Bray R, De Laat M, Godinot X, Ugarte A, Walker R. The hidden dimensions of poverty: international participatory research [Internet]. Oxford University, ATD Fourth World; 2019. Available from: https://www.atdfourthworld.org/wp-content/uploads/sites/5/2019/05/Dim_ Pauvr_eng_FINAL.pdf

13. Stange KC, Ferrer RL. The paradox of primary care. Ann Fam Med. 2009; 7(4):293-9.

\section{ENDEREÇO PARA CORRESPONDÊNCIA}

E-mail: paulabroeiro@gmail.com

https://orcid.org/0000-0002-5013-6171 